\title{
Organ transplantation crosses species divide
}

Mice can be cured of diabetes with pancreatic cells grown in rats.

Mouse models of diabetes transplanted with pancreatic cells grown in rats maintain normal blood sugar levels for more than a year - nearly the entire lifespan of a mouse. "This is a lifetime cure for diabetes," says stem-cell researcher Hiromitsu Nakauchi at the University of Tokyo and Stanford University, who led the study (Nature 542, 191-196; 2017). "Our research provides a proof-of-concept for the ultimate goal of making human organs in animals."

For patients with fatal organ disease, replacing their failing organs with functioning ones remains the only form of treatment. But increasing demand for transplantable organs has met with a critical donor shortage, with many patients dying before receiving the lifesaving operation. More than 115,000 patients in the United States are currently on the organ waiting list, of which close to 100,000 anticipate a kidney, and 3,000 a pancreas.

Bioengineering offers the promise of an unlimited supply of organs. One approach scientists are taking is to coax pluripotent stem cells, which can give rise to any cell type in the body, into miniature organs in a dish. But these in vitro techniques are still far from mimicking the complex anatomy of an adult organ. An alternative method seeks to grow human organs in a closely related host animal. Nakauchi's team is the first to show that this is a viable therapeutic option.

In a previous study, Nakauchi's lab successfully grew a rat pancreas in a mouse host, but the organ turned out to be mousesized. "It was ten times smaller than in a wild-type rat," explains first author of the current paper, Tomoyuki Yamaguchi, at the University of Tokyo. Mice are generally smaller in size than rats. The organ was so small that the researchers could not extract sufficient pancreatic cells to treat rats.

Nakauchi and Yamaguchi decided to reverse the experiment, generating a mouse pancreas in a rat host. They first bioengineered pancreas-free rats by mutating a crucial gene involved in pancreatic development in rat embryos. They then isolated embryonic cells from the apancreatic rats

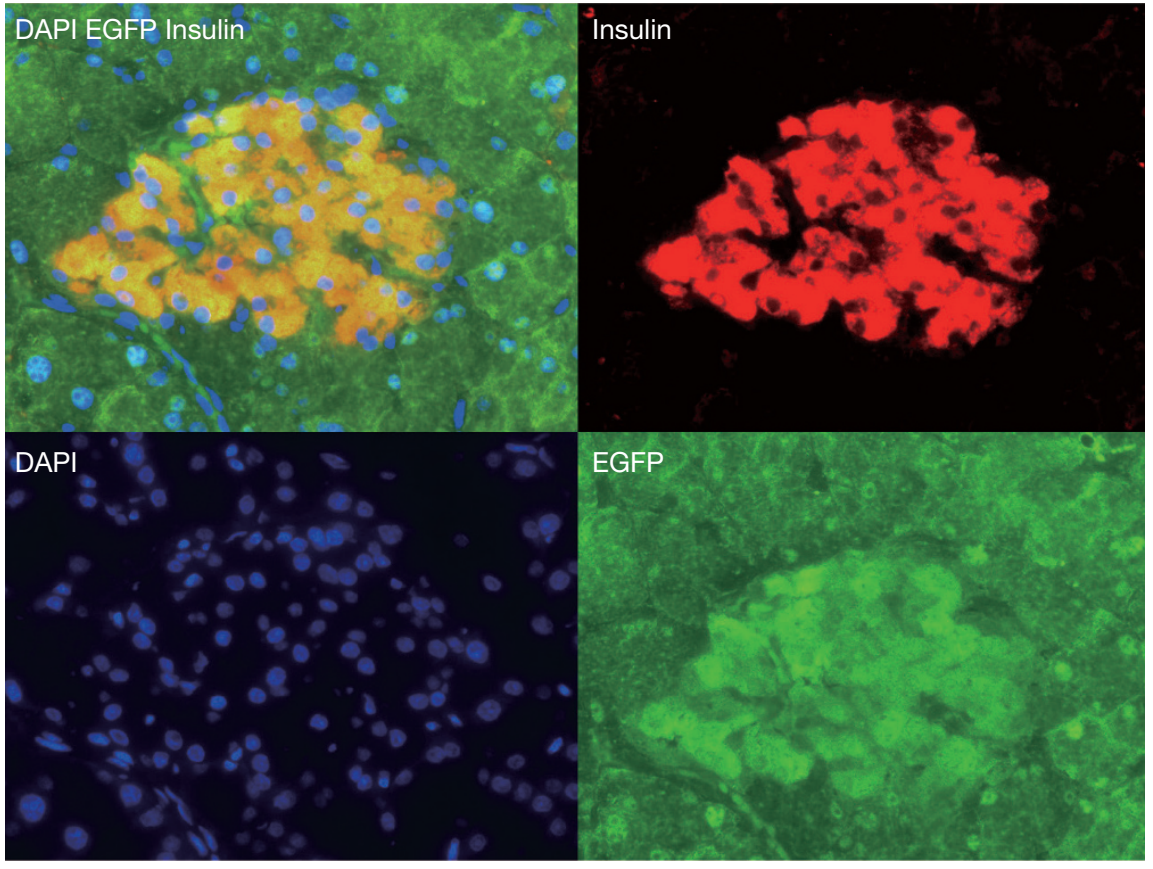

Immunofluorescence image of pancreatic islet from a chimeric $P d \times 1^{\mathrm{mu} / \mathrm{mu}}$ rat showing insulin-producing cells.

and injected them with mouse pluripotent stem cells. The next generation of rats had rat-sized pancreases, some made entirely of mouse cells, and others mixed with rat cells.

Cell clusters scattered throughout the pancreas, known as islets, produce hormones that regulate blood glucose levels in the body. In type 1 diabetes, these glucose control centers do not function properly. Nakauchi and Yamaguchi tested the therapeutic potential of their harvested islets by transplanting them into mouse models of type 1 diabetes. The mouse immune systems were suppressed for five days postoperation, to avoid triggering an immune response from residual rat cells in the transplanted tissue. But no further immunosuppression was needed as the genetic makeup of donor and host mouse cells were identical.

The results were positive. The mice recovered their ability to regulate glucose, maintaining normal levels for the entire study period of 370 days. "This level of functionality has never been shown in in vitro generated islets," says Nakauchi. "Our data are by far the best." A similar response was observed in mice that received islet transplants from normal mouse donors, but those that received rat islets could not control their glucose levels.

A major worry with applying this technique in pig-to-human organ donation is the possibility of exposing humans to infectious viruses originating in pigs. But Nakauchi maintains that no such infection has been observed in any study to date.

In any case, says Nakauchi, scientists first need to prove that the technology can work in larger animal models that are evolutionarily more distant. "Once we succeed in generating human organs in pigs or sheep, then we will focus on the safety issues."

His team has already grown apancreatic pigs, which could host monkey or human cells. And a group led by Juan Carlos Izpisua Belmonte at the Salk Institute for Biological Studies in California have created human-pig hybrid embryos.

"We would like to generate human organs in pigs, and then use the organs to treat patients with severe type 1 diabetes, liver failure or kidney failure," says Yamaguchi. Smriti Mallapaty 